
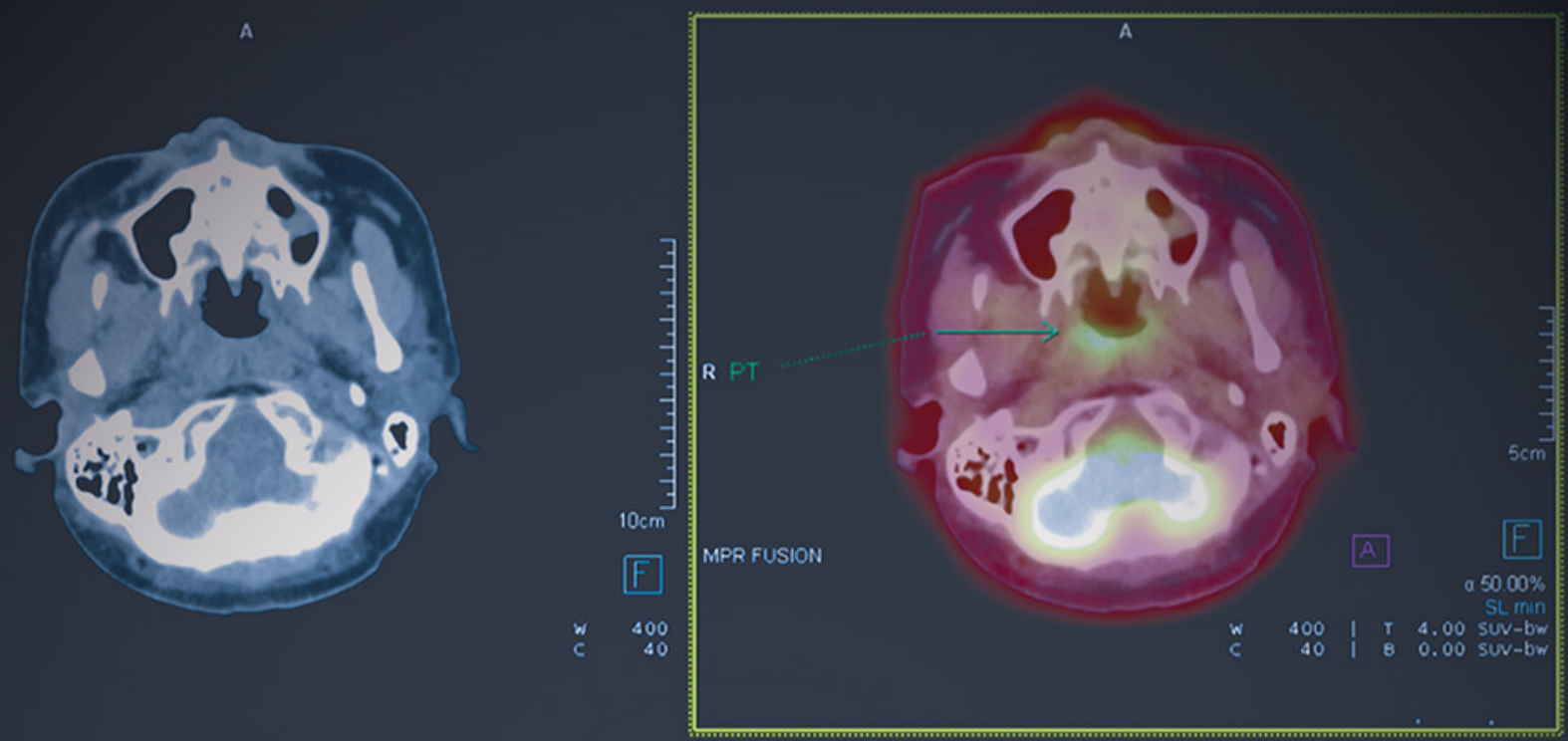

An Evidence-Based Approach to the Management of

Nasopharyngeal Cancer

From Basic Science to Clinical Presentation and Treatment

Edited by

Baharudin Abdullah

Anusha Balasubramanian

Norhafiza Mat Lazim

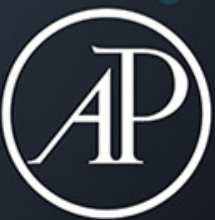




\section{An Evidence-Based Approach to the Management of Nasopharyngeal Cancer}


This page intentionally left blank 


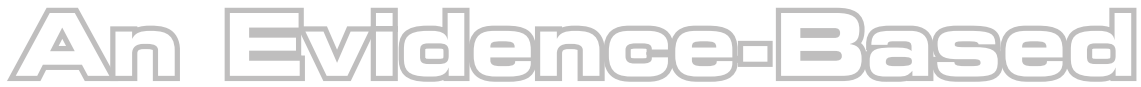

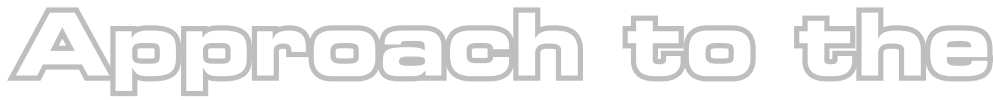

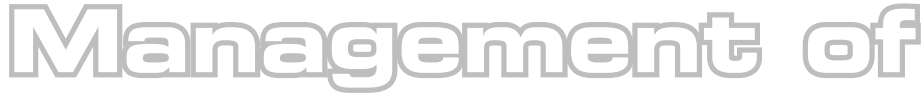

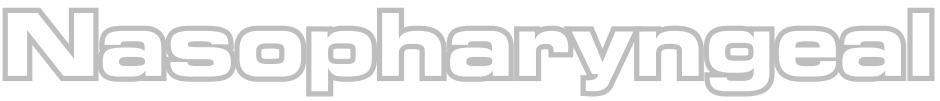

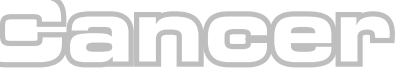 \\ From Basic Science to Clinical Presentation and Treatment}

Edited by

\section{Baharudin Abdullah}

Senior Consultant, Department of Otorhinolaryngology - Head and Neck Surgery, School of Medical Sciences, Universiti Sains Malaysia, Kubang Kerian, Kelantan, Malaysia

\section{Anusha Balasubramanian}

Head and Neck Unit, The Royal Marsden Hospital, Chelsea, London, United Kingdom

Norhafiza Mat Lazim

Consultant Otorhinolaryngology-Head and Neck Surgery, Department of Otorhinolaryngology-Head and Neck Surgery, School of Medical Sciences, Universiti Sains Malaysia, Health Campus, Kubang Kerian, Kelantan, Malaysia
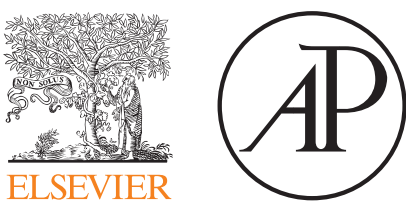

ACADEMIC PRESS

An imprint of Elsevier 
Academic Press is an imprint of Elsevier

125 London Wall, London EC2Y 5 AS, United Kingdom

525 B Street, Suite 1650, San Diego, CA 92101, United States

50 Hampshire Street, 5th Floor, Cambridge, MA 02139, United States

The Boulevard, Langford Lane, Kidlington, Oxford OX ${ }_{5}{ }_{1} G B$, United Kingdom

Copyright (C) 2020 Elsevier Inc. All rights reserved.

No part of this publication may be reproduced or transmitted in any form or by any means, electronic or mechanical, including photocopying, recording, or any information storage and retrieval system, without permission in writing from the publisher. Details on how to seek permission, further information about the Publisher's permissions policies and our arrangements with organizations such as the Copyright Clearance Center and the Copyright Licensing Agency, can be found at our website: www.elsevier.com/permissions.

This book and the individual contributions contained in it are protected under copyright by the Publisher (other than as may be noted herein).

\section{Notices}

Knowledge and best practice in this field are constantly changing. As new research and experience broaden our understanding, changes in research methods, professional practices, or medical treatment may become necessary.

Practitioners and researchers must always rely on their own experience and knowledge in evaluating and using any information, methods, compounds, or experiments described herein. In using such information or methods they should be mindful of their own safety and the safety of others, including parties for whom they have a professional responsibility.

To the fullest extent of the law, neither the Publisher nor the authors, contributors, or editors, assume any liability for any injury and/or damage to persons or property as a matter of products liability, negligence or otherwise, or from any use or operation of any methods, products, instructions, or ideas contained in the material herein.

\section{British Library Cataloguing-in-Publication Data}

A catalogue record for this book is available from the British Library

\section{Library of Congress Cataloging-in-Publication Data}

A catalog record for this book is available from the Library of Congress

ISBN: 978-0-12-814403-9

For Information on all Academic Press publications

visit our website at https://www.elsevier.com/books-and-journals

Publisher: Stacy Masucci

Acquisitions Editor: Rafael Teixeira

Editorial Project Manager: Tracy Tufaga

Production Project Manager: Poulouse Joseph

Cover Designer: Matthew Limbert

Typeset by MPS Limited, Chennai, India 


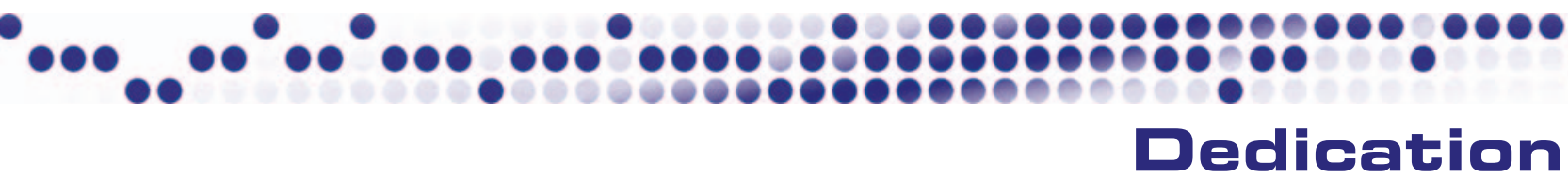

\section{Baharudin Abdullah}

To my loving wife, Dr. Eka Sumianti, who has always been patient with me and my two beautiful girls, Clarissa Andini and Carneisha Aleeya. I will always love and cherish you all until the end of time.

\section{Anusha Balasubramanian}

To my beloved parents, husband, godparents, teachers, patients, colleagues and friends; thank you for guiding me through the path of life, I remain eternally grateful and indebted to you. God bless.

\section{Norhafiza Mat Lazim}

I would like to dedicate this book to my caring husband Associate Professor Dr. Zul Izhar Mohd Ismail, who has been very supportive through the journey of completing the chapters for this book. My sincere gratitude also goes to my three beloved children, Arieff Iskandar, Adry Zahrin, and Alyssa Yasmin, who have been my endless motivation for me. I am deeply grateful and blessed to have continuous support and encouragement from my other immediate family members, friends and close colleagues at work. 



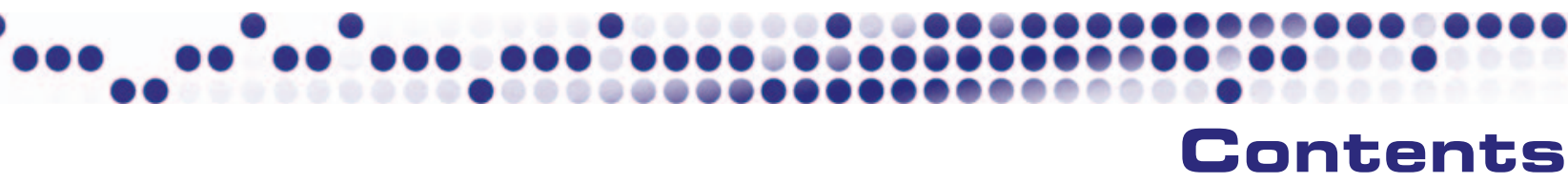

List of Contributors

xiii

About the editors

xvii

Acknowledgment

xix

Chapter 1 Introduction 1

William Ignace Wei and Raymond K. Tsang

Historical aspect 1

Geographical distribution and familial clustering 3

Association with Epstein-Barr virus 4

Improvement in treatment outcomes in the last five decades 5

Conclusion 8

$\begin{array}{ll}\text { References } & 8\end{array}$

Chapter 2 Risk factors and etiopathogenesis of nasopharyngeal carcinoma

Norhafiza Mat Lazim and Baharudin Abdullah

Introduction

Interaction between the risk factors and pathogenesis of nasopharyngeal carcinoma

Association between stages of nasopharyngeal carcinoma at diagnosis with risk factors of nasopharyngeal carcinoma

Implications for the management of nasopharyngeal carcinoma

Epstein-Barr virus as a screening biomarker

How to identify and reduce risk

Conclusion

References

Further reading 


\section{Contents}

\section{Chapter 3 Genetics of nasopharyngeal carcinoma and molecular signaling pathways}

Murtala B. Abubakar and Siew Hua Gan

Genomic and epigenetic landscapes of nasopharyngeal carcinoma

List of abbreviations

References 50

Further reading

Chapter 4 Surgical anatomy of the nasopharynx

Ing Ping Tang, Prepageran Narayanan and Zul Izhar Mohd. Ismail

Introduction

Surgical anatomy of nasopharynx

Important surgical adjacent structures

Conclusion

References

\section{Chapter 5 Pathology classification of nasopharyngeal} carcinoma

Sharifah Emilia Tuan Sharif, Norzaliana Zawawi, Aidy Irman Yajid, Norasnieda Md Shukri and Irfan Mohamad

Introduction

The evolved history of nasopharyngeal carcinoma pathology classifications

WHO classifications

Histomorphological features of nasopharyngeal carcinoma

Ancillary studies

Nasopharyngeal carcinoma diagnostic problems

Conclusions

Acknowledgment

References 


\section{Contents}

Chapter 6 Clinical presentation of nasopharyngeal carcinoma 93

Marlinda Adham, Norhafiza Mat Lazim and Roman Carlos

Introduction

Four groups of clinical signs and symptoms of nasopharyngeal carcinoma

Sign and symptoms related to extension of disease out of the nasopharynx

Conclusion

References 106

Further reading

\section{Chapter 7 Assessment and staging of nasopharyngeal} carcinoma

Karman Ka Ying Leung and Michael Chi Fai Tong

Assessment

Staging

Summary and concluding remarks

References

Chapter 8 Laboratory investigation for nasopharyngeal carcinoma diagnosis

Mai Abdel Haleem Abu Salah, Hasnan Jaafar and

Chan Yean Yean

Introduction

Epstein-Barr virus serology

Histopathological evaluation

Application of brush cytology versus tissue biopsy

Conclusion

References

\section{Chapter 9 Open surgical salvage procedures for nasopharyngeal carcinoma}

Raymond K. Tsang

Introduction 


\section{Contents}

Assessment of nodal failures of nasopharyngeal

carcinoma for surgical salvage

Neck dissection for salvaging nodal failures in

nasopharyngeal carcinoma

Reirradiation of the neck with brachytherapy after neck

dissection

References

Chapter 10 Radiation therapy and chemotherapy in early and advanced nasopharyngeal cancer

Francis Ho and Gokula Kumar Appalanaido

Principles of radiation therapy

Intensity modulated radiotherapy plan approval

Types of radiation therapy specifically including intensity modulated radiotherapy in nasopharyngeal cancer

Role of radiation in early disease

Management of the neck

Complications of radiation

Reirradiation in nasopharyngeal cancer recurrence

Treatment of locally advanced disease

Systemic therapy

Role of radiation in metastatic disease

Conclusion

References

Further reading

Chapter 11 Role of endoscopic endonasal surgery in recurrent nasopharyngeal carcinoma: endoscopic endonasal transpterygoid nasopharyngectomy

Ing Ping Tang and Prepageran Narayanan

Introduction

Patient selection

Preoperative workup 


\section{Contents}

Surgical technique

Postoperative care and complications

Conclusion

References

Chapter 12 Nasopharyngeal carcinoma screening and prevention programs

Anusha Balasubramanian and Norhafiza Mat Lazim

Introduction to screening

Rationale for screening

Epstein-Barr virus serology as a screening tool

242

Epstein-Barr virus DNA for screening

244

Nasendoscopy

244

Circulating biomarkers

245

Effectiveness of screening

Rationale for prevention

High-risk group

Education programs targeting dietary habits

Low-risk groups

General population-based programs

Conclusion

References

Further reading

\section{Chapter 13 Survival rates and quality of life of} nasopharyngeal carcinoma patients

Norhafiza Mat Lazim, Giacomo Spinato, Kahairi Abdullah and Paolo Boscolo-Rizzo

Introduction 255

Survival rates of nasopharyngeal carcinoma patients 256

Effect of patient-related factors

Effect of treatment-related factors 


\section{Contents}

Effect of addition of chemotherapy on quality of life 266

Effect of surgery on quality of life 267

Quality of life as prognostic marker in patients with nasopharyngeal cancer

Conclusion

References

Further reading

\section{Chapter 14 New developments in nasopharyngeal cancer}

Ing Bing Tan, Norhafiza Mat Lazim, Sharon D. Stoker and Maarten A. Wildeman

Early diagnosis by Epstein-Barr virus biomarkers

Imaging modality of nasopharyngeal cancer

Treatment of nasopharyngeal cancer

Primary treatment for nasopharyngeal cancer

New emerging treatments in nasopharyngeal cancer

Local failures in nasopharyngeal cancer

Surgical treatment of recurrent or persistent nasopharyngeal cancer

Photodynamic therapy in nasopharyngeal cancer

Photodynamic therapy versus surgery and radiotherapy for local failures of nasopharyngeal cancer

Immunotherapy for nasopharyngeal cancer

Conclusion

References

Further reading 


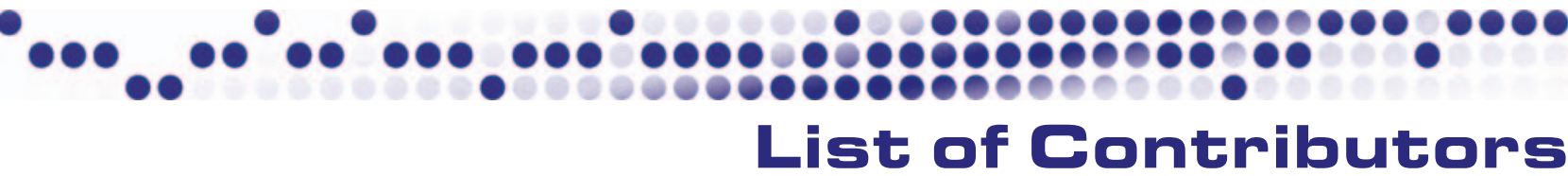

Baharudin Abdullah Department of Otorhinolaryngology-Head and Neck Surgery, School of Medical Sciences, Universiti Sains Malaysia, Health Campus, Kubang Kerian, Kelantan, Malaysia

Kahairi Abdullah Department of ORL-Head and Neck Surgery, Kuliyyah of Medicine, International Islamic University Malaysia, Kuantan, Pahang

Murtala B. Abubakar Department of Physiology, Faculty of Basic Medical Sciences, College of Health Sciences, Usmanu Danfodiyo University, Sokoto, Nigeria; Human Genome Centre, School of Medical Sciences, Universiti Sains Malaysia, Kubang Kerian, Malaysia

Marlinda Adham Otorhinolaryngology Head\&Neck Department, Faculty of Medicine Universitas Indonesia, Dr. Cipto Mangunkusumo Hospital, Jakarta, Indonesia

Gokula Kumar Appalanaido Advanced Medical \& Dental Institute, Universiti Sains Malaysia, Pulau Pinang, Malaysia

Anusha Balasubramanian Head and Neck Unit, The Royal Marsden Hospital NHS Foundation Trust, Chelsea, London, United Kingdom

Paolo Boscolo-Rizzo Department of Surgery, Oncology and Gastroenterology, section of Oncology and Immunology, University of Padova, Padova, Italy

Roman Carlos Department of Otolaryngology-Head and Neck Surgery, Hospital Universitario Reina Sofía, Cordoba, Spain

Siew Hua Gan School of Pharmacy, Monash University Malaysia, Jalan Lagoon Selatan, Bandar Sunway, Malaysia

Francis Ho National University Cancer Institute, National University Health System, Singapore

Zul Izhar Mohd. Ismail Department of Anatomy, School of Medical Sciences, Universiti Sains Malaysia, Health Campus, Kubang Kerian, Kelantan, Malaysia

Hasnan Jaafar Department of Pathology, School of Medical Sciences, Universiti Sains Malaysia, Health Campus, Kota Bharu, Malaysia 
Norhafiza Mat Lazim Department of Otorhinolaryngology-Head and Neck Surgery, School of Medical Sciences, Universiti Sains Malaysia, Health Campus, Kubang Kerian, Kelantan, Malaysia

Karman Ka Ying Leung Department of Otorhinolaryngology, Head and Neck Surgery, The Chinese University of Hong Kong, Shatin, P.R. China

Irfan Mohamad Department of Otorhinolaryngology-Head and Neck Surgery, School of Medical Sciences, Universiti Sains Malaysia, Health Campus, Kubang Kerian, Kelantan, Malaysia

Prepageran Narayanan Department of ORL-HNS, Faculty of Medicine, University of Malaya, Kuala Lumpur, Malaysia

Mai Abdel Haleem Abu Salah Department of Medical Microbiology and Parasitology, School of Medical Sciences, Universiti Sains Malaysia, Health Campus, Kota Bharu, Malaysia

Sharifah Emilia Tuan Sharif Department of Pathology, School of Medical Sciences, Universiti Sains Malaysia, Kubang Kerian, Malaysia; Hospital Universiti Sains Malaysia, Kubang Kerian, Malaysia

Norasnieda Md Shukri Department of Otorhinolaryngology-Head and Neck Surgery, School of Medical Sciences, Universiti Sains Malaysia, Health Campus, Kubang Kerian, Kelantan, Malaysia

Giacomo Spinato Department of Neurosciences, Section of Otolaryngology and Regional Centre of Head and Neck Cancer, University of Padova, Treviso, Italy; Department of Surgery, Oncology and Gastroenterology, section of Oncology and Immunology, University of Padova, Padova, Italy

Sharon D. Stoker Department of Otorhinolaryngology-Head and Neck Surgery, AmsterdamUMC, The Netherlands

Ing Bing Tan Department of Otolaryngology-Head and Neck Surgery, Maastricht University Medical Center, Maastricht, The Netherlands; Department of Otolaryngology-Head and Neck Surgery, Gadjah Mada University, Yogyakarta, Indonesia; Department of Head and Neck surgery of The Netherlands Cancer Institute, Amsterdam, The Netherlands

Ing Ping Tang Department of ORL-HNS, Faculty of Medicine \& Health Sciences, Universiti Malaysia Sarawak, Sarawak, Malaysia

Michael Chi Fai Tong Department of Otorhinolaryngology, Head and Neck Surgery, The Chinese University of Hong Kong, Shatin, P.R. China

Raymond K. Tsang Department of Surgery, The University of Hong Kong, Pokfulam, Hong Kong SAR, China 
William Ignace Wei Department of Surgery, Hong Kong Sanitorium and Hospital, Happy Valley, Hong Kong SAR, China

Maarten A. Wildeman Department of Otorhinolaryngology Alrijne Hospital, Leiderdorp, The Netherlands

Aidy Irman Yajid Department of Pathology, School of Medical Sciences, Universiti Sains Malaysia, Kubang Kerian, Malaysia; Hospital Universiti Sains Malaysia, Kubang Kerian, Malaysia

Chan Yean Yean Department of Medical Microbiology and Parasitology, School of Medical Sciences, Universiti Sains Malaysia, Health Campus, Kota Bharu, Malaysia

Norzaliana Zawawi Department of Pathology, School of Medical Sciences, Universiti Sains Malaysia, Kubang Kerian, Malaysia; Hospital Universiti Sains Malaysia, Kubang Kerian, Malaysia; School of Dental Sciences, Universiti Sains Malaysia, Kubang Kerian, Malaysia 


\title{
Role of endoscopic endonasal surgery in recurrent nasopharyngeal carcinoma: endoscopic endonasal transpterygoid nasopharyngectomy
}

\author{
Ing Ping Tang ${ }^{1}$ and Prepageran Narayanan² \\ ${ }^{1}$ Department of ORL-HNS, Faculty of Medicine \& Health Sciences, Universiti Malaysia Sarawak, \\ Sarawak, Malaysia ${ }^{2}$ Department of ORL-HNS, Faculty of Medicine, University of Malaya, \\ Kuala Lumpur, Malaysia
}

\section{Introduction}

The primary treatment for untreated nasopharyngeal carcinoma (NPC) is radiotherapy (RT) alone for early-stage lesion and concurrent chemo-radiation for advanced tumor. Suarez et al. (2010), Ma et al. (2001), and Lin, Liang, Jan, Jiang, and Lin (2004) reported that incidence of local recurrence was approximately $8 \%$ to $58 \%$. You et al. (2015) mentioned locally recurrent NPC can be treated with salvage reirradiation or surgery. Ridge (1993) recommended that resection of locally discrete recurrent NPC should be considered unless the patient is unfit for surgery. Tumors that persist or recur after primary radiotherapy have demonstrated significant resistance to radiotherapy. Surgery should be considered for patients presenting with residual or recurrent tumor after radiotherapy or chemoradiotherapy, and for patients with glandular or mesenchymal differentiated tumors that are poorly responsive to radiotherapy as mentioned by Al-Sheibani et al. (2011). Furthermore, high dose reirradiation may still result in severe complications like osteoradionecrosis, brain necrosis, radiation induced myelitis, hypopituitarism and trismus as described by To et al. (2002) and Chang et al. (2000).

Nasopharyngectomy is a well-established surgical procedure for salvaging locally recurrent NPC; via various surgical approaches, that is, midfacial degloving, 
transpalatal, transmaxillary, maxillary swing, or transmandibular as mentioned by King, Ku, Mok, and Teo (2000). The common complications for open nasopharyngectomy are middle ear effusion, palatal fistula, nasal regurgitation and trismus.

Ideally, a surgical approach to the nasopharynx should provide adequate visualization of the tumor margins, allow complete oncologic resection with negative margins, allow the possibility to extend the resection margins if necessary, and allow the identification and protection of important neurovascular structures. In addition, it should avoid facial scarring or deformity, preserve neurologic and masticatory functions, and facilitate the reconstruction of the surgical defect. Over the past decade, the evolution of these approaches has incorporated endoscopic endonasal techniques to complement conventional skull base approaches, and in certain patients, as the sole approach.

Endoscopic endonasal transpterygoid nasopharyngectomy (EETN) has then emerged as a viable treatment option for locally recurrent NPC with minimal invasiveness, avoiding morbidity from external approaches and the absence of facial scar. A literature review by Emanuelli et al. (2014) showed that endoscopic method attained a higher negative surgical margin of $93.75 \%$ than external approach (71.6\%).

Radical neck dissection or modified radical neck dissection should be performed for regional recurrence of NPC at the neck, provided there is no distant metastasis. Either unilateral or bilateral neck dissections are performed depending on the extent of the nodal disease.

\section{Patient selection}

Patient selection is perhaps the most important aspect of effectively treating patients with EETN. Generally speaking, patients categorized as rT1, those categorized as rT2 with minimal parapharyngeal extension, and select patients categorized as rT3 (Involvement of floor of sphenoid sinus) can be treated with EETN as described by Chen et al. (2012). Exclusion criteria for patients are based on disease factors and patient factors. Disease factors include significant parapharyngeal space extension, internal carotid artery (ICA) involvement, cavernous sinus with multiple cranial nerves involvement, brain parenchymal involvement and presence of distant metastasis. Patient factors include patients who are medically unfit to tolerate surgery and undergo general anesthesia.

\section{Preoperative workup}

All patients who undergo EETN will have thorough preoperative workup. The preoperative workup includes clinical factors, radiological factors, and pathological factors.

For clinical factors, patients require a thorough medical examination including nasopharyngoscopy to delineate the tumor extension. The patient's full medical 
status must also be investigated to determine eligibility for general anesthesia. For radiological factors, radiological examination is essential to ascertain the extent of the primary tumor, assess for regional lymph node involvement, and rule out distant metastatic disease. Investigational techniques include computed tomography and magnetic resonance imaging (MRI), and occasionally positron emission tomography scan. For pathological factors, histopathological examination of the suspected local recurrence or residual tumor and type of tumor must be confirmed before any surgical intervention.

Special attention has to be given to ascertain the relationship of the tumor to the parapharyngeal and petrous segments of the ICA. Generally, patients with tumors that are encasing the ICA and, extensive dura or intradural involvement are not suitable candidates for EETN. Other important preoperative preparations depending on the individual case include computed tomography angiography (CTA) with computed tomography and MRI fusion for intraoperative navigation. An acoustic Doppler ultrasound probe can complement the surgical navigation device for the identification of critical vessels, especially ICA. The preferred prophylactic perioperative antibiotic regimen should include a thirdgeneration cephalosporin with cerebral spinal fluid penetration.

\section{urgical technique}

The surgery is done under general anesthesia with the patient lying in supine position. The nasal cavities are decongested with Moffett's solution for 30 minutes as illustrated by Benjamin, Wong, and Choa (2004). The solution contains $1 \mathrm{~mL}$ adrenaline $1: 1000,2 \mathrm{~mL}$ of $10 \%$ cocaine, $4 \mathrm{~mL}$ of $8.4 \%$ sodium bicarbonate, mixed together with $13 \mathrm{~mL}$ of water for injection. Following general anesthesia, there is widespread vasodilation, hence producing hyperemic mucosa. A topical decongestant of Moffett's solution could reduce nasal blood flow, optimizing the surgical field. Infiltration of both middle turbinates and nasal septum with a solution of lidocaine $1 \%$ and epinephrine 1/100,000 enhances the hemostasis. Surgery proceeds via a purely endoscopic endonasal approach using a 0 - and 30-degree rod lens endoscope. A fundamental premise of the endonasal endoscopic approach is that two surgeons work concomitantly, using a bimanual, three-/four-handed technique via both nostrils and nasal cavities. This facilitates dynamic visualization as well as bimanual dissection, which is vital for depth perception, traction, countertraction, and for maintenance of a blood-free surgical field.

\section{Sinonasal corridor}

Firstly, surgery is initiated by enlarging the natural sinonasal corridor ipsilateral to the lesion by removing the inferior half of the right middle turbinate and completing an uncinectomy, large midmeatal nasomaxillary window, and anterior and posterior ethmoidectomies. This increases the working space and 
Figure 11.1

Right medial maxillectomy performed as part of the transpterygoid approach.

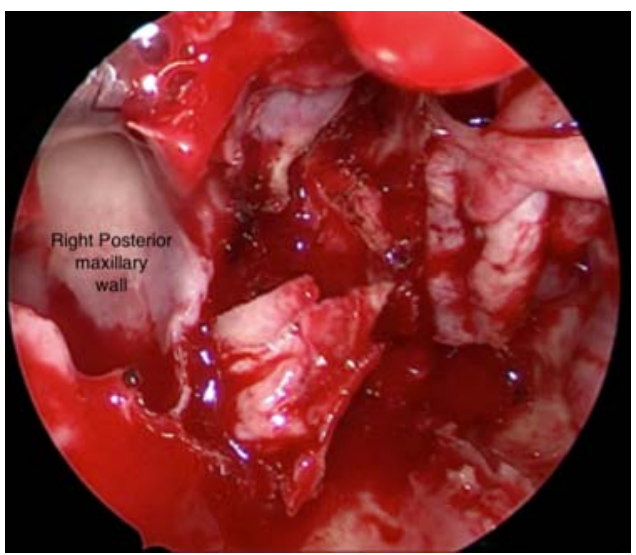

expose the entire posterior wall of the antrum. A medial maxillectomy is performed to expose the entire height of the posterior wall of the maxillary sinus and to allow an extended dissection of the pterygopalatine fossa. This medial maxillectomy is limited anteriorly by the nasolacrimal duct, which acts like a fulcrum point, preventing free movement of the scope laterally. Endoscopic Denker's approach (also known as the Sturman-Canfield approach) can be performed to further increase the lateral angle of exposure and optimize instrument maneuverability. Endoscopic Denker's approach is a procedure to remove the piriform aperture, as well as the anterior maxillary wall, until the lateral wall of the antrum is in direct and full view especially viewing the entire infratemporal fossa (Fig. 11.1).

Additional lateral control is obtained by bringing the instruments from the contralateral side of the nose through a posterior septectomy. A generous posterior bony septectomy allows a bimanual technique traversing both sides of the nasal cavity. This extensive posterior septectomy allows visualization of the entire posterior wall of the maxillary sinus using a 0 -degree endoscope that crosses over to the contralateral side of the nose.

\section{Nasoseptal flap}

The Hadad-Bassagasteguy nasoseptal flap (HBF) should be harvested from the contralateral side of the tumor as illustrated by Al-Sheibani et al. (2011). It is critical to harvest the HBF from the contralateral side because its pedicle and proximal blood supply would be surrendered ipsilateral to the transpterygoid dissection. Later, a Caicedo reverse flap is transposed from the contralateral side to cover the HBF donor defect as illustrated by Prosser, Figueroa, Carrau, Ong, and Solares (2011). Clinical harvesting of these septal flaps presumes that the tumor does not involve this area. If tumor involves the nasal septum, other vascularized flaps can be considered (Figs. 11.2-11.5). 
An Evidence-Based Approach to the Management of Nasopharyngeal Cancer

Figure 11.2

Creating HadadBassagasteguy nasoseptal flap (HBF).

Figure 11.3

Posterior

septectomy.

Figure 11.4

Creating Caicedo's flap.
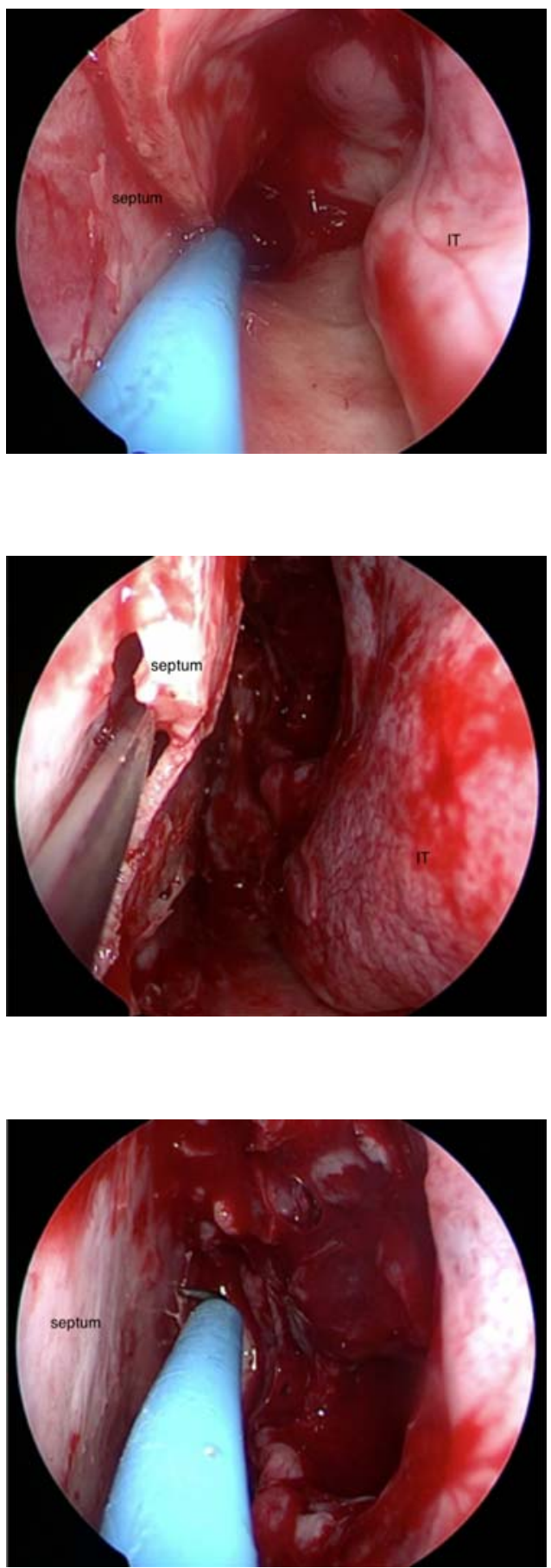
Figure 11.5

Two nostrils and four hands technique after removing posterior septum.

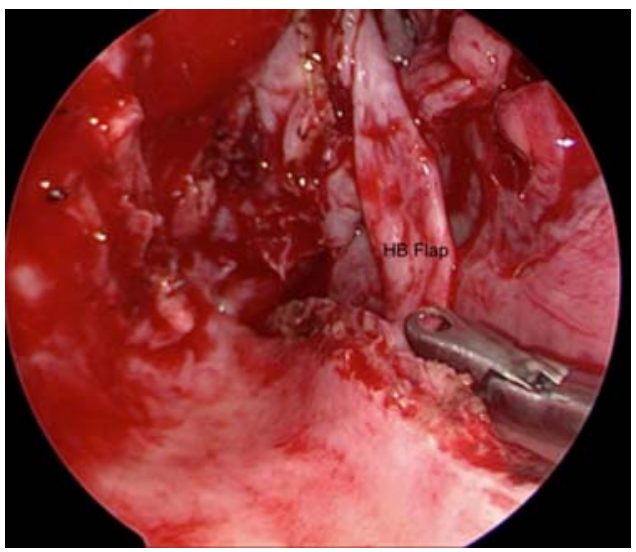

\section{Inferior sphenoidectomy}

The anterior face of the sphenoid sinus is often opened early during the approach, enlarging the sphenoid ostium after completing the ethmoidectomies. As the superior part of the sphenoid crest is removed, the sella turcica's floor and intersinus and intrasinus septations, as well as the lateral walls of the sphenoid sinus, come into direct view. Prior to removing the inferior component of the sphenoid rostrum, its mucoperiosteum is dissected laterally and inferiorly to identify key anatomic landmarks as this area comprises the pedicle of the HBF as described by Hadad et al. (2006).

The lateral walls of the sphenoid and the medial pterygoid plates (lateral wall of the posterior choana) form a vertical strut that intersects the floor of the sphenoid sinus. The junction of the vertical medial pterygoid plate with the horizontal floor of the sphenoid sinus forms a wedge-shaped area ("pterygoid wedge") that contains both the vidian canal and the palatovaginal canal. After complete removal of the vomer, the intersinus septum, and the sphenoid sinus floor, the sphenoidectomy should extend superiorly to be in plane with the roof of the nose and laterally to be in plane with the laminae papyracea bilaterally as described by Caicedo-Granados et al. (2010). Complete removal of the sphenoid sinus floor is performed until the cavity is flush with the clivus (Fig. 11.6).

\section{Pterygopalatine fossa dissection}

Dissection of the soft tissue contents of the pterygopalatine fossa is a prerequisite for the transpterygoid approach. After exposing the entire posterior maxillary sinus wall via medial maxillectomy, the sphenopalatine and posterior nasal arteries are divided at the level of the sphenopalatine foramen. Removal of the posterior wall of the antrum and the ascending process of the palatine bone exposes the pterygopalatine fossa with its intrinsic and bordering foramina and fissures as mentioned by Hosseini et al. (2012). 
Figure 11.6

Drilling anterior wall and floor of the sphenoid sinus.

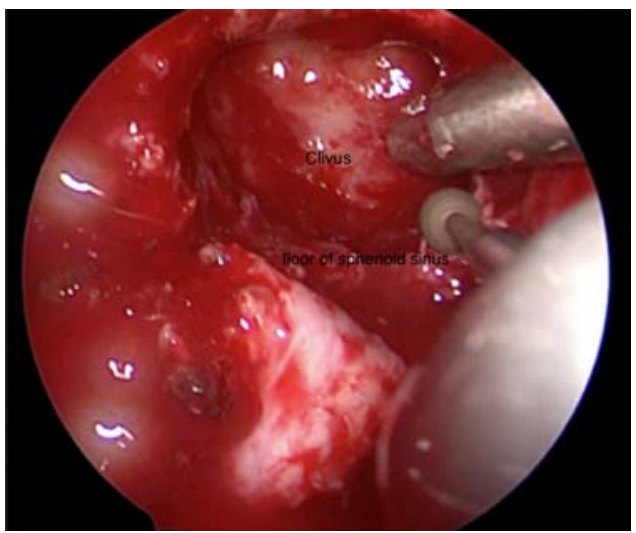

\section{Transpterygoid dissection}

A transpterygoid dissection starts with the identification of the vidian nerve proximal to the pterygopalatine ganglion, as it exits from the vidian canal. The vidian nerve can be localized following the palatovaginal canal laterally or by drilling its canal starting at the pterygoid wedge. The vidian nerve, within the pterygoid canal, courses toward the second genu of the ICA between the horizontal and vertical segments. For the most part, the vidian canal remains inferior to the second genu of the ICA; therefore initial drilling in a 3-9 o'clock orientation, helps to prevent injury to the ICA. In some cases, the superior aspect of the canal is covered just with a very thin bone or may even be dehiscent, thus exposing the nerve in the floor of the sphenoid sinus.

The maxillary division of the trigeminal nerve passes through the foramen rotundum as it courses from Meckel's cave into the pterygopalatine fossa as described by Fortes et al. (2008). The maxillary nerve can also be identified in its canal coursing the lateral wall of the sphenoid sinus. The pharyngeal end of the Eustachian tube or torus tubarius is just posterior to the pterygoid process. Removal of the pterygoid process exposes the cartilaginous Eustachian tube. The parapharyngeal segment of the ICA is posterior to the Eustachian tube in most of the cases.

All these landmarks are crucial to identify during the transpterygoid approach before tumor extirpation (Figs. 11.7 and 11.8).

\section{Tumor extirpation}

Tumor removal begins by marking out at least $1 \mathrm{~cm}$ margin if technically possible around the tumor. The mucosal cuts are made with needlepoint electrocautery, which helps with hemostasis. The superior and posterior dissection occurs by elevating the mucoperiosteum from the floor of the sphenoid sinus and the clivus posteriorly. Monteiro and Witterick (2014) mentioned the dissection proceeds inferiorly to the level of the soft palate, until the prevertebral musculature deep to 
Figure 11.7

Drilling pterygoid base and medial pterygoid plate.

Figure 11.8

Resecting of cartilage part of eustachian tube.
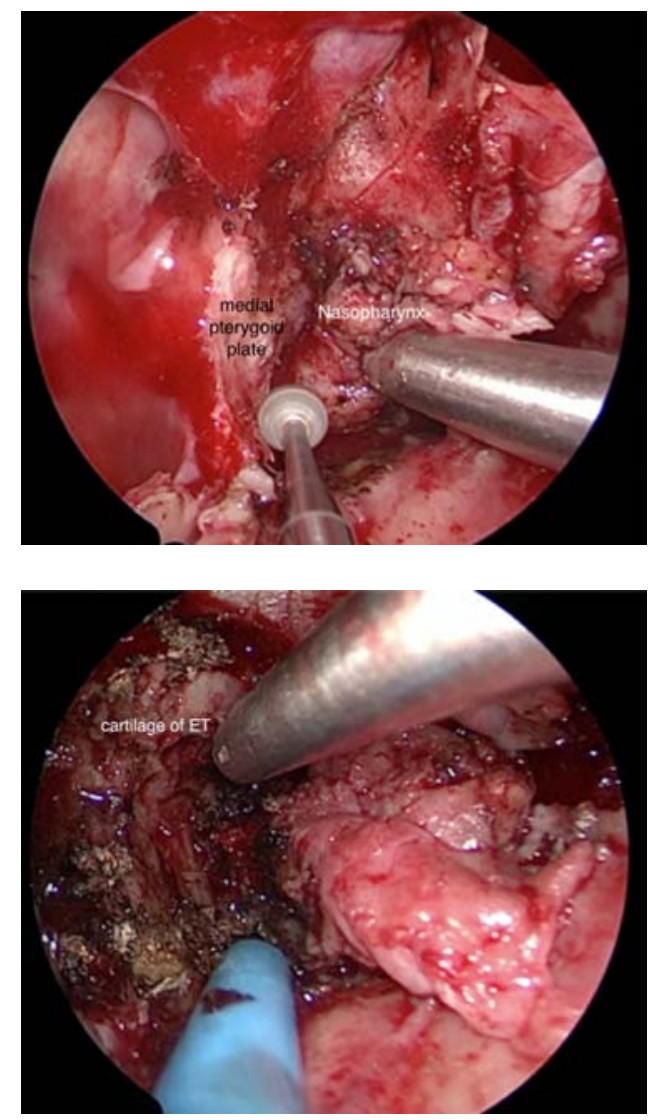

the pharyngobasilar fascia and prevertebral fascia is encountered. Electrocautery or Kerrison rongeurs both can be used, and are effective at removing portions of the prevertebral muscle and fascia, as these structures are quite resilient. The muscles are included in the en-bloc resection if involved with malignancy.

Laterally, the medial pterygoid plate and pterygoid process are exposed, above which lies the sinus of Morgagni, through which passes the Eustachian tube and tensor veli palatine muscle. These structures can be excised along with the levator palatini muscle to expose the parapharyngeal tissues as mentioned by Kassam et al. (2009). The mucosa is elevated off the base of the foramen lacerum and continues anteriorly toward the posterior aspect of the fossa of Rosenmuller. The Eustachian tube cartilage laterally is identified and included in the specimen. As the dissection proceeds laterally, the Doppler probe is used to map out the ICA, thereby reducing the likelihood of inadvertent injury. Following complete tumor removal, margin status is confirmed by sending circumferential and deep margins for frozen section analysis (Figs. 11.9 and 11.10). 
Figure 11.9

Resecting

posteriorly at prevertebral muscle.

Figure 11.10

Final view of resection of right fossa of Rosenmuller (FOR) of nasopharynx.
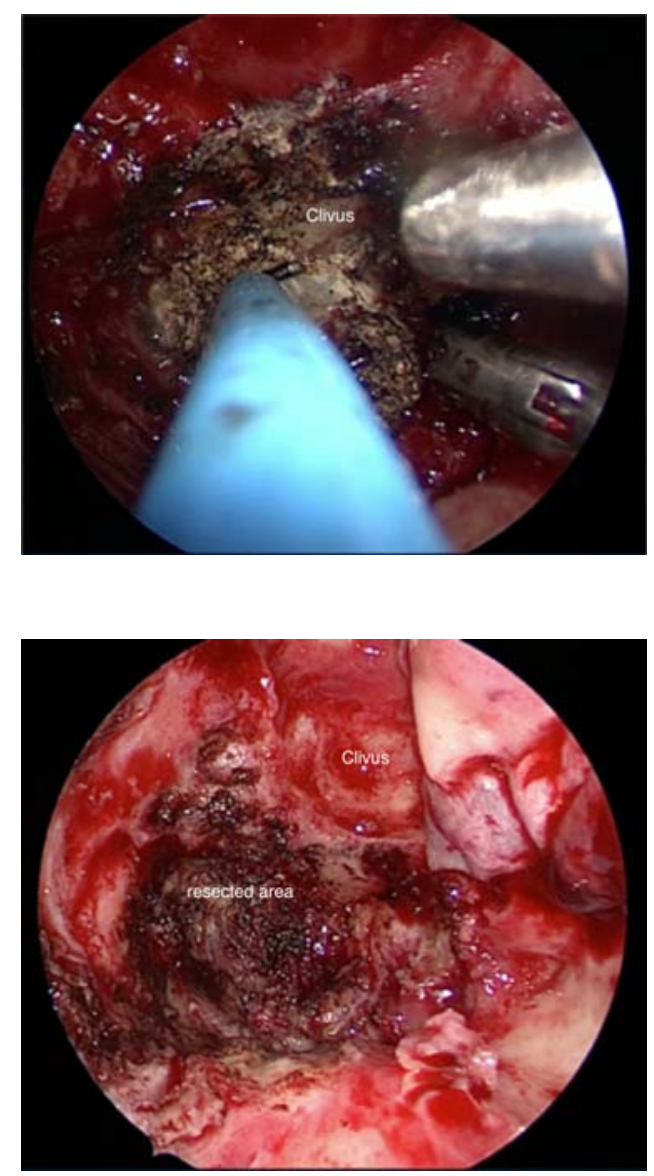

\section{Nasopharyngeal reconstruction}

Yip et al. (2013) described reconstruction with a vascularized pedicle flap at the nasopharyngeal defect facilitates the healing of the defect, resists irradiation, and protects the ICA against exposure and blowout. The nasoseptal flap or HBF is rotated into the nasopharyngeal defect. The edges of the flap are allied well to cover the bare area especially exposed bony portion at the clivus. Absorbable gelatin sponges are placed on the flap, and a Foley catheter is used to support the nasoseptal flap against the nasopharyngeal defect. In cases where the nasoseptal flap is unavailable, a lateral nasal wall flap can be harvested for reconstruction. Other reconstructive options include healing by secondary intention or the use of regional flaps such as pedicled temporoparietal fascia flap (Figs. 11.11 and 11.12).

Following reconstruction, the nasal cavity is thoroughly inspected for hemostasis followed by careful suction of the nasopharyngeal cavity. The patient is then 


\section{Figure 11.11}

Placing of HadadBassagasteguy flap (HBF) to the

resected area.

Figure 11.12

Catheter tube with balloon to hold the flap at place.
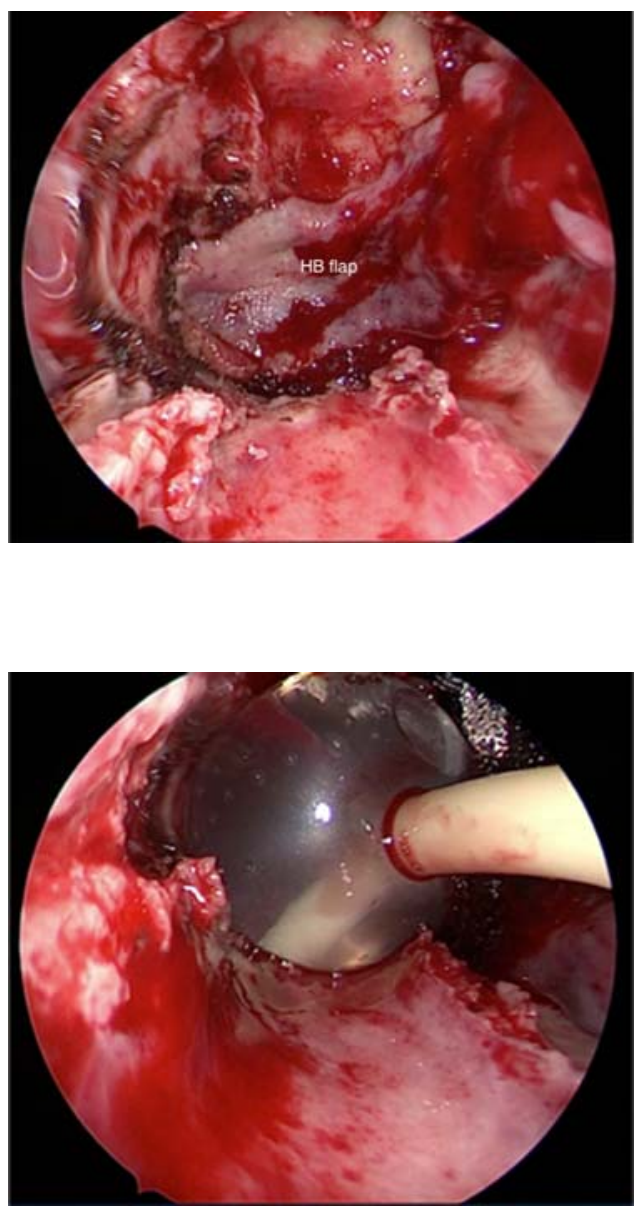

reversed from general anesthesia and transferred to the postoperative care unit for recovery. The patient is admitted to a standard ward unit and is usually discharged from hospital within $2-3$ days.

\section{Postoperative care and complications}

Patients are generally seen every $2-3$ weeks to undergo endoscopic examination. During these clinic procedures, decrusting is performed, paying careful attention not to disrupt the nasoseptal flap or any other flaps that are laid on. Patients are asked to liberally use sodium bicarbonate mixed with mupirocin 
nasal rinses 3-4 times per day. They may experience a significant amount of postoperative crusting requiring frequent endoscopic debridement until reepithelialization occurs. This process can take up to 3 or more months, even with a nasoseptal flap. Postoperative headache is a common complaint following EETN due to exposed bone. Exposed bone covered with a pedicled nasoseptal flap reduces this incidence significantly.

Serous otitis media is another common complication encountered in the postoperative period. This can be managed by myringotomy with tympanostomy tube insertion or amplification with hearing aids.

Postoperative epistaxis can occur secondary to bleeders that are not secured during the surgery especially when the nasoseptal flap is not in used, and the bleeding is from the posterior septal artery. Other possible surgical sequelae include xerophthalmia secondary to injury of the vidian nerve, numbness in the V2 distribution, skull base injury including cerebrospinal fluid leak, and injury to the ICA in either its parapharyngeal, petrous, or clival portions as described by Valentine and Wormald (2011).

\section{Conclusion}

In conclusion, EETN is a feasible approach for the surgical treatment of selected primary and recurrent nasopharyngeal tumors. The surgical technique requires a trained and experienced team with specialized instruments. This technique shows relatively low morbidity with promising preliminary outcomes and local control of the disease that is comparable to conventional techniques.

\section{References}

Al-Sheibani, S., Zanation, A. M., Carrau, R. L., Prevedello, D. M., Prokopakis, E. P., McLaughlin, N., ... Kassam, A. B. (2011). Endoscopic endonasal transpterygoid nasopharyngectomy. The Laryngoscope, 121, 2081-2089.

Benjamin, E., Wong, D. K., \& Choa, D. (2004). 'Moffett's' solution: A review of the evidence and scientific basis for the topical preparation of the nose. Clinical Otolaryngology and Allied Sciences, 29(6), 582-587.

Caicedo-Granados, E., Carrau, R., Snyderman, C. H., Prevedello, D., Fernandez-Miranda, J., \& Gadner, P. (2010). Reverse rotation flap for reconstruction of donor site after vascular pedicled nasoseptal flap in skull-base surgery. The Laryngoscope, 120, 1550-1552.

Chang, J. T. C., See, L. C., Liao, C. T., Ng, S. H., Wang, C. H., Chen, I. H., ... Hong, J. H. (2000). Locally recurrent nasopharyngeal carcinoma. Radiotherapy and Oncology, 54, $135-142$.

Chen, M. Y., Wang, S. L., Zhu, Y. L., Sgen, G. P., Qiu, F., \& Luo, D. H. (2012). Use of a posterior pedicle nasal septum and floor mucoperiosteum flap to resurface the nasopharynx after endoscopic nasopharyngectomy for recurrent nasopharyngeal carcinoma. Head \& Neck, 34, 1383-1388. 
Emanuelli, E., Albu, S., Cazzador, D., Pedruzzi, B., Babighian, G., \& Martini, A. (2014). Endoscopic surgery for recurrent undifferentiated nasopharyngeal carcinoma. The Journal of Craniofacial Surgery, 25, 1003-1008.

Fortes, F. S., Sennes, L. U., Carrau, R. L., Brito, R., Ribas, G. D., \& Yasuda, A. (2008). Endoscopic anatomy of the pterygopalatine fossa and transpterygoid approach: Development of a surgical instruction model. The Laryngoscope, 118, 44-49.

Hadad, G., Bassagasteguy, L., Carrau, R. L., Mataza, J. C., Kassam, A., \& Snyderman, C. H. (2006). A novel reconstructive technique after endoscopic expanded endonasal approaches: Vascular pedicle nasoseptal flap. The Laryngoscope, 116, 1882-1886.

Hosseini, S. M., Razfar, A., Carrau, R. L., Prevedello, D. M., Fernande-Miranda, J., \& Zanation, A. (2012). Endonasal transpterygoid approach to the infratemporal fossa: Correlation of endoscopic and mutiplanar CT anatomy. Head \& Neck, 34, 313-320.

Kassam, A. B., Prevedello, D. M., Carrau, R. L., Snyderman, C. H., Gardner, P., \& Osawa, S. (2009). The front door to Meckel's cave: An anteromedial corridor via expanded endoscopic endonasal approach-technical considerations and clinical series. Neurosurgery, 64, 71-83.

King, W. W., Ku, P. K., Mok, C. O., \& Teo, P. M. (2000). Nasopharyngectomy in the treatment of recurrent nasopharyngeal carcinoma: A twelve-year experience. Head \& Neck, 22, 215-222.

Lin, J. C., Liang, W. M., Jan, J. S., Jiang, R. S., \& Lin, A. C. (2004). Another way to estimate outcome of advanced nasopharyngeal carcinoma-is concurrent chemoradiotherapy adequate? International Journal of Radiation Oncology, Biology, Physics, 60, 156-164.

Ma, J., Mai, H. Q., Hong, M. H., Min, H. Q., Mao, Z. D., Cui, N. J., ... Mo, H. Y. (2001). Results of a prospective randomized trial comparing neoadjuvant chemotherapy plus radiotherapy with radiotherapy alone in patients with locoregionally advanced nasopharyngeal carcinoma. Journal of Clinical Oncology, 19, 1350-1357.

Monteiro, E., \& Witterick, I. (2014). Endoscopic nasopharyngectomy: Patient selection and surgical execution. Operative Techniques in Otolaryngology, 25, 284-288.

Prosser, J. D., Figueroa, R., Carrau, R. I., Ong, Y. K., \& Solares, C. A. (2011). Quatificative analysis of endoscopic endonasal approaches to the infra temporal fossa. The Laryngoscope, 121, 1601-1605.

Ridge, J. A. (1993). Squamous cancer of the head and neck: Surgical treatment for local and regional recurrence. Seminars in Oncology, 20, 419-429.

Suarez, C., Rodrigo, J. P., Rinaldo, A., Langendijk, J. A., Shaha, A. R., \& Ferlito, A. (2010). Current treatment options for recurrent nasopharyngeal cancer. European Archives of Otorhino-laryngology, 267, 1811-1824.

To, E. W., Lai, E. C., Cheng, J. H., Pang, P. C., Williams, M. D., \& Teo, P. M. (2002). Nasopharyngectomy for recurrent nasopharyngeal carcinoma: A review of 31 patients and prognostic factors. The Laryngoscope, 112, 1877-1882.

Valentine, R., \& Wormald, P. J. (2011). Carotid artery injury after endonasal surgery. Otolaryngologic Clinics of North America, 44, 1059-1079.

Yip, J., Macdonald, K. I., Lee, J., Wittlerick, I. J., Zadeh, G., \& Gentili, F. (2013). The inferior turbinate flap in skull base reconstruction. Journal of Otolaryngology-Head \& Neck Surgery, 31, 42-46.

You, R., Zou, X., Hua, Y. J., Han, F., Li, L., Zhao, C., ... Chen, M. Y. (2015). Salvage endoscopic nasopharyngectomy is superior to intensity-modulated radiation therapy for local recurrence of selected T1-T3 nasopharyngeal carcinoma-a case-matched comparison. Radiotherapy and Oncology, 115, 399-406. 